Georgian Mathematical Journal

Volume 12 (2005), Number 1, 1-10

\title{
TWO-STEP SYSTEMS FOR G-H-RELAXED PSEUDOCOCOERCIVE NONLINEAR VARIATIONAL PROBLEMS BASED ON PROJECTION METHODS
}

\author{
RAVI P. AGARWAL, DONAL O'REGAN, RAM U. VERMA
}

\begin{abstract}
The approximation-solvability of a generalized system of nonlinear variational inequalities (SNVI) involving relaxed pseudococoercive mappings, based on the convergence of a system of projection methods, is presented. The class of relaxed pseudococoercive mappings is more general than classes of strongly monotone and relaxed cocoercive mappings. Let $K_{1}$ and $K_{2}$ be nonempty closed convex subsets of real Hilbert spaces $H_{1}$ and $H_{2}$, respectively. The two-step SNVI problem considered here is as follows: find an element $\left(x^{*}, y^{*}\right) \in H_{1} \times H_{2}$ such that $\left(g\left(x^{*}\right), g\left(y^{*}\right)\right) \in K_{1} \times K_{2}$ and

$$
\begin{array}{ll}
\left\langle S\left(x^{*}, y^{*}\right), g(x)-g\left(x^{*}\right)\right\rangle \geq 0 & \forall g(x) \in K_{1}, \\
\left\langle T\left(x^{*}, y^{*}\right), h(y)-h\left(y^{*}\right)\right\rangle \geq 0 & \forall h(y) \in K_{2},
\end{array}
$$

where $S: H_{1} \times H_{2} \rightarrow H_{1}, T: H_{1} \times H_{2} \rightarrow H_{2}, g: H_{1} \rightarrow H_{1}$ and $h: H_{2} \rightarrow H_{2}$ are nonlinear mappings.

2000 Mathematics Subject Classification: 49J40, 65B05.

Key Words and phrases: Cocoercive mapping, relaxed cocoercive mapping, relaxed pseudococoercive variational inequality, two-step system of variational inequalities, convergence of projection methods.
\end{abstract}

\section{INTRODUCTION}

The convergence analysis for projection/projection type methods has been frequently applied to the approximate solvability of problems arising in several fields of applied mathematical sciences, including complementarity problems, convex quadratic programming, optimization theory, and variational problems. There exists an enormous amount of literature on the approximate solvability of several classes of variational inequalities and related problems in different space settings. Since the notion of relaxed pseudococoercivity is more general than strong monotonicity and relaxed cocoercivity, the obtained results generalize investigations on strongly monotone and relaxed cocoercive types of variational inequality problems based on projection methods. Verma [6] has introduced and studied the approximation solvability of a two-step system of strongly monotone nonlinear variational inequalities in a Hilbert space setting, while Fang and Huang [2] have introduced and studied a system of variational inclusions involving $H$-monotone and strongly monotone mappings based on an algorithm using the resolvent operator technique. Recently, Rhoades and Verma [5] and Kassay and Kolumban [3] have studied systems of variational inequalities in 
different space settings. In this paper, we consider, based on a system of projection methods, the approximation solvability of a system of nonlinear relaxed pseudococoercive variational inequalities on Hilbert spaces. For more details on variational inequalities and projection methods, we refer the reader to [1-12].

Let $H_{1}$ and $H_{2}$ be two real Hilbert spaces with the inner product $\langle$,$\rangle and$ norm $\|\cdot\|$ on both $H_{1}$ and $H_{2}$. Let $S: H_{1} \times H_{2} \rightarrow H_{1}$ and $T: H_{1} \times H_{2} \rightarrow H_{2}$ be any mappings on $H_{1} \times H_{2}$ and, $K_{1}$ and $K_{2}$ be closed convex subsets of $H_{1}$ and $H_{2}$, respectively. We consider problem tied with a system of nonlinear variational inequalities (abbreviated as SNVI) as follows: determine an element $\left(x^{*}, y^{*}\right) \in H_{1} \times H_{2}$ such that $\left(g\left(x^{*}\right), h\left(y^{*}\right)\right) \in K_{1} \times K_{2}$ and

$$
\begin{array}{ll}
\left\langle S\left(x^{*}, y^{*}\right), g(x)-g\left(x^{*}\right)\right\rangle \geq 0 & \forall g(x) \in K_{1}, \\
\left\langle T\left(x^{*}, y^{*}\right), h(y)-h\left(y^{*}\right)\right\rangle \geq 0 & \forall h(y) \in K_{2},
\end{array}
$$

where $g: H_{1} \rightarrow H_{1}$ and $h: H_{2} \rightarrow H_{2}$ are any mappings.

When $g \equiv I$ and $h \equiv I$ (identity), we have the SNVI problem: find an element $\left(x^{*}, y^{*}\right) \in K_{1} \times K_{2}$ such that

$$
\begin{array}{ll}
\left\langle S\left(x^{*}, y^{*}\right), x-x^{*}\right\rangle \geq 0 & \forall x \in K_{1}, \\
\left\langle T\left(x^{*}, y^{*}\right), y-y^{*}\right\rangle \geq 0 & \forall y \in K_{2} .
\end{array}
$$

The SNVI (1.1)-(1.2) problem is equivalent to the following projection formulas

$$
\begin{aligned}
& g\left(x^{*}\right)=P_{K}\left[g\left(x^{*}\right)-\rho S\left(x^{*}, y^{*}\right)\right] \text { for } \rho>0, \\
& h\left(y^{*}\right)=Q_{K}\left[h\left(y^{*}\right)-\eta T\left(x^{*}, y^{*}\right)\right] \text { for } \eta>0,
\end{aligned}
$$

where $P_{K}$ is the projection of $H_{1}$ onto $K_{1}$ and $Q_{K}$ is the projection of $H_{2}$ onto $K_{2}$.

The SNVI (1.1)-(1.2) problem extends the nonlinear variational inequality problem: determine an element $x^{*} \in K$ such that

$$
\left\langle T\left(x^{*}, x^{*}\right), x-x^{*}\right\rangle \geq 0 \quad \forall x \in K .
$$

Now we need to recall the following auxiliary results, most frequently used in the context of approximation-solvability of nonlinear variational inequality problems based on iterative procedures.

Lemma $1.1([3])$. For an element $z \in H$ and for a nonempty closed convex subset $K$ of $H$, we have

$$
x \in K \quad \text { and } \quad\langle x-z, y-x\rangle \geq 0 \quad \forall y \in K \quad \text { if and only if } x=P_{K}(z) .
$$

Lemma 1.2. An element $u$ is a solution to (1.5) iff $u=P_{K}[u-\lambda T(u, u)]$ for all $\lambda>0$.

Definition 1.1. A mapping $T: H \rightarrow H$ is called:

(i) monotone if for each $x, y \in H$, we have

$$
\langle T(x)-T(y), x-y\rangle \geq 0 ;
$$


(ii) $r$-strongly monotone if for each $x, y \in H$, we have

$$
\langle T(x)-T(y), x-y\rangle \geq r\|x-y\|^{2} \text { for a constant } r>0 ;
$$

(iii) $r$-expansive if

$$
\|T(x)-T(y)\| \geq r\|x-y\|
$$

(iv) expansive if

$$
\|T(x)-T(y)\| \geq\|x-y\| ;
$$

(v) $s$-Lipschitz continuous (or Lipschitzian) if there exists a constant $s \geq 0$ such that

$$
\|T(x)-T(y)\| \leq s\|x-y\| \quad \forall x, y \in H
$$

(vi) $\mu$-cocoercive $[1,6]$ if for each $x, y \in H$, we have

$$
\langle T(x)-T(y), x-y\rangle \geq \mu\|T(x)-T(y)\|^{2} \text { for a constant } \mu>0 .
$$

Clearly, every $\mu$-cocoercive mapping $T$ is $(1 / \mu)$-Lipschitz continuous.

We can easily see that the following implications on monotonicity, strong monotonicity and expansiveness hold:

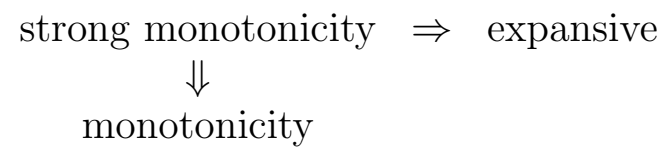

Definition 1.2. A mapping $T: H$ is said to be:

(i) relaxed $g$-cocoercive if there exists a constant $g>0$ such that

$$
\langle T(x)-T(y), x-y\rangle \geq(-\gamma)\|T(x)-T(y)\|^{2} \quad \forall x, y \in H ;
$$

(ii) relaxed $(\gamma, r)$-cocoercive if there exist constants $g, r>0$ such that

$$
\langle T(x)-T(y), x-y\rangle \geq(-\gamma)\|T(x)-T(y)\|^{2}+r\|x-y\|^{2} .
$$

On the top of that we have the implication

$$
\text { strong } r \text {-monotonicity } \Rightarrow \text { relaxed } \gamma \text {-r-cocoercivity. }
$$

Definition 1.3. A mapping $T: H \rightarrow H$ is said to be:

(i) relaxed $(g, \gamma, r)$-cocoercive if there exist positive constants $\gamma$ and $r$ such that

$\langle T(x)-T(y), g(x)-g(y)\rangle \geq(-\gamma)\|T(x)-T(y)\|^{2}+r\|g(x)-g(y)\|^{2} \quad \forall x, y \in H$, where $g: H \rightarrow H$ is any mapping on $H$;

(ii) relaxed $(g, \gamma, r)$-pseudococoercive if there exist positive constants $\gamma$ and $r$ such that

$$
\begin{gathered}
\langle T(y), g(x)-g(y)\rangle \geq 0 \\
\Rightarrow\langle T(x), g(x)-g(y)\rangle \geq(-\gamma)\|T(x)-T(y)\|^{2}+r\|g(x)-g(y)\|^{2} \quad \forall x, y \in H,
\end{gathered}
$$

where $g: H \rightarrow H$ is any mapping on $H$.

Proposition 1.1. If $T: H \rightarrow H$ is $r$-strongly monotone, then $T$ is relaxed $(\gamma, r)$-cocoercive for $\gamma, r>0$, while the converse may not be true in general. 
Example 1.1. Consider a $\mu$-Lipschitz continuous mapping $T: H \rightarrow H$ on $H$. Then we have:

(i) $I-T$ is $(1-\mu)$-strongly monotone for $0<\mu<1$.

(ii) $I-T$ is relaxed $(\gamma,(1-\mu))$-cocoercive for $\gamma>0$ and $0<\mu<1$, where $I$ is the identity mapping on $H$.

\section{Systems of Projection Methods}

In this section we present the convergence of projection methods in the context of the approximation-solvability of the SNVI (1.1)-(1.2).

Algorothm 2.1. For an arbitrarily chosen initial point $\left(g\left(x^{0}\right), h\left(y^{0}\right)\right) \in$ $K_{1} \times K_{2}$, compute sequences $\left\{g\left(x^{k}\right)\right\}$ and $\left\{h\left(y^{k}\right)\right\}$ such that

$$
\begin{aligned}
& \left.g\left(x^{k+1}\right)=\left(1-a^{k}\right) g\left(x^{k}\right)+a^{k} P_{K}\left[g\left(x^{k}\right)-\rho S\left(x^{k}, y^{k}\right)\right)\right], \\
& h\left(y^{k+1}\right)=\left(1-a^{k}\right) h\left(y^{k}\right)+a^{k} Q_{K}\left[h\left(y^{k}\right)-\eta T\left(x^{k}, y^{k}\right)\right],
\end{aligned}
$$

where $P_{K}$ is the projection of $H_{1}$ onto $K_{1}$ and $Q_{K}$ is the projection of $H_{2}$ onto $K_{2}, \rho, \eta>0$ are constants, and

$$
0 \leq a^{k} \leq 1 \quad \text { and } \quad \sum_{k=0}^{\infty} a^{k}=\infty .
$$

When $g \equiv I$ and $h \equiv I$, Algorithm 2.1 reduces to

Algorothm 2.2. For an arbitrarily chosen initial point $\left(x^{0}, y^{0}\right) \in K_{1} \times K_{2}$, compute sequences $\left\{x^{k}\right\}$ and $\left\{y^{k}\right\}$ such that

$$
\begin{aligned}
& \left.x^{k+1}=\left(1-a^{k}\right) x^{k}+a^{k} P_{K}\left[x^{k}-\rho S\left(x^{k}, y^{k}\right)\right)\right], \\
& y^{k+1}=\left(1-a^{k}\right)\left(y^{k}\right)+a^{k} Q_{K}\left[y^{k}-\eta T\left(x^{k}, y^{k}\right)\right],
\end{aligned}
$$

where $P_{K}$ is the projection of $H_{1}$ onto $K_{1}$ and $Q_{K}$ is the projection of $H_{2}$ onto $K_{2}, \rho, \eta>0$ are constants, and

$$
0 \leq a^{k} \leq 1 \quad \text { and } \quad \sum_{k=0}^{\infty} a^{k}=\infty .
$$

We now present, based on Algorithm 2.1, the approximation-solvability of the SNVI (1.1)-(1.2) problem involving relaxed $(g, \gamma, r)$-pseudococoercive and relaxed $(h, \lambda, s)$-pseudococoercive mappings in the Hilbert space setting.

Theorem 2.1. Let $H_{1}$ and $H_{2}$ be two real Hilbert spaces, and $K_{1}$ and $K_{2}$ be nonempty closed convex subsets of $H_{1}$ and $H_{2}$, respectively. Let $S: H_{1} \times H_{2} \rightarrow$ $H_{1}$ be relaxed $(g, \gamma, r)$-pseudococoercive and $(g, \mu)$-Lipschitz continuous in the first variable, and let $S$ be $(h, \nu)$-Lipschitz continuous in the second variable. Let $\mathrm{T}: \mathrm{H}_{1} \times \mathrm{H}_{2} \rightarrow \mathrm{H}_{2}$ be relaxed $(h, \lambda, s)$-pseudococoercive and $(h, \beta)$-Lipschitz continuous in the second variable, and let $T$ be $(g, \tau)$-Lipschitz continuous in the first variable. Let $g: H_{1} \rightarrow H_{1}$ and $h: H_{2} \rightarrow H_{2}$ be expansive mappings. Let $\|(u, v)\|_{x}$ denote the norm on $\mathrm{H}_{1} \times \mathrm{H}_{2}$ defined by

$$
\|(u, v)\|_{x}=\|u\|+\|v\| \quad \text { for }(u, v) \in H_{1} \times H_{2},
$$


where $\|\cdot\|$ denotes the norm on both $H_{1}$ and $H_{2}$.

In addition, let $\delta=\max \{\theta+\eta \tau, \sigma+\rho \nu\}$, where

$$
\begin{gathered}
\theta=\left[1-2 \rho r+2 \rho \gamma \mu^{2}+\rho+\left(\rho \mu^{2} / 2\right)+(\rho \mu)^{2}\right]^{1 / 2}, \\
\sigma=\left[1-2 \eta s+2 \eta \lambda \beta^{2}+\eta+\left(\eta \beta^{2} / 2\right)+(\eta \beta)^{2}\right]^{1 / 2} \\
\theta+\eta \tau<1, \quad \sigma+\rho \nu<1 .
\end{gathered}
$$

Suppose that the following assumptions hold:

(i) the element $\left(g\left(x^{*}\right), h\left(y^{*}\right)\right) \in K_{1} \times K_{2}$ forms a solution to the SNVI (1.1) -(1.2) problem;

(ii) sequences $\left\{g\left(x^{k}\right)\right\}$ and $\left\{h\left(y^{k}\right)\right\}$ are generated by Algorithm 2.1;

(iii) the sequence $\left\{a^{k}\right\}$ satisfies

$$
0 \leq a^{k} \leq 1 \quad \text { and } \quad \sum_{k=0}^{\infty} a^{k}=\infty ;
$$

(iv) $\left\langle S\left(x^{*}, y^{k}\right), g\left(x^{k}\right)-g\left(x^{*}\right)\right\rangle \geq 0$;

(v) $\left\langle T\left(x^{k}, y^{*}\right), h\left(y^{k}\right)-h\left(y^{*}\right)\right\rangle \geq 0$.

Then the sequence $\left\{\left(g\left(x^{k}\right), h\left(y^{k}\right)\right)\right\}$ converges to $\left(g\left(x^{*}\right), h\left(y^{*}\right)\right)$.

Corollary 2.1. Let $H_{1}$ and $H_{2}$ be two real Hilbert spaces and $K_{1}$ and $K_{2}$ be nonempty closed convex subsets of $H_{1}$ and $H_{2}$, respectively. Let $S: K_{1} \times$ $K_{2} \rightarrow H_{1}$ be $(g, r)$-strongly monotone and $(g, \mu)$-Lipschitz continuous in the first variable, and let $S$ be $(h, \nu)$-Lipschitz continuous in the second variable. Let $T: K_{1} \times K_{2} \rightarrow H_{2}$ be $(h, s)$-strongly monotone and $(h, \beta)$-Lipschtz continuous in the second variable, and let $T$ be $(g, \tau)$-Lipschitz continuous in the first variable. Let $g: K_{1} \rightarrow K_{1}$ and $h: K_{2} \rightarrow K_{2}$ be expansive mappings. Let $\|\cdot\|_{x}$ denote the norm on $H_{1} \times H_{2}$ defined by

$$
\|(u, v)\|_{x}=\|u\|+\|v\| \quad \text { for } \quad(u, v) \in H_{1} \times H_{2},
$$

where $\|\cdot\|$ denotes the norm on both $\mathrm{H}_{1}$ and $\mathrm{H}_{2}$.

In addition, let $d=\max \{\theta+\eta \tau, \sigma+\rho \nu\}$, where

$$
\begin{aligned}
& \theta=\left[1-2 \rho r+(\rho \mu)^{2}\right]^{1 / 2}, \\
& \sigma=\left[1-2 \eta s+(\eta \beta)^{2}\right]^{1 / 2}, \\
& \theta+\eta \tau<1, \quad \sigma+\rho \nu<1 .
\end{aligned}
$$

Suppose that the following assumptions hold:

(i) the element $\left(g\left(x^{*}\right), h\left(y^{*}\right)\right) \in K_{1} \times K_{2}$ forms a solution to the SNVI (1.1)-(1.2) problem;

(ii) sequences $\left\{g\left(x^{k}\right)\right\}$ and $\left\{h\left(y^{k}\right)\right\}$ are generated by Algorithm 2.1;

(iii) The sequence $\left\{a^{k}\right\}$ satisfies

$$
0 \leq a^{k} \leq 1 \quad \text { and } \quad \sum_{k=0}^{\infty} a^{k}=\infty .
$$

Then the sequence $\left\{\left(g\left(x^{k}\right), h\left(y^{k}\right)\right)\right\}$ converges to $\left(g\left(x^{*}\right), h\left(y^{*}\right)\right)$. 
Proof of Theorem 2.1. Since $\left(g\left(x^{*}\right), h\left(y^{*}\right)\right)$ is a solution of the SNVI (1.1)-(1.2) problem, it follows that

$$
g\left(x^{*}\right)=P_{K}\left[g\left(x^{*}\right)-\rho S\left(x^{*}, y^{*}\right)\right] \text { and } h\left(y^{*}\right)=Q_{K}\left[h\left(y^{*}\right)-\eta T\left(x^{*}, y^{*}\right)\right] .
$$

Applying Algorithm 2.1, we have

$$
\begin{aligned}
\| g\left(x^{k+1}\right) & -g\left(x^{*}\right)\|=\|\left(1-a^{k}\right) g\left(x^{k}\right)+a^{k} P_{K}\left[g\left(x^{k}\right)-\rho S\left(x^{k}, y^{k}\right)\right] \\
& -\left(1-a^{k}\right) g\left(x^{*}\right)-a^{k} P_{K}\left[g\left(x^{*}\right)-S\left(x^{*}, y^{*}\right)\right] \| \\
\leq & \left(1-a^{k}\right)\left\|g\left(x^{k}\right)-g\left(x^{*}\right)\right\|+a^{k} \| P_{K}\left[g\left(x^{k}\right)-\rho S\left(x^{k}, y^{k}\right)\right] \\
& -P_{K}\left[g\left(x^{*}\right)-\rho S\left(x^{*}, y^{*}\right)\right] \| \\
\leq & \left(1-a^{k}\right)\left\|g\left(x^{k}\right)-g\left(x^{*}\right)\right\|+a^{k}\left\|g\left(x^{k}\right)-g\left(x^{*}\right)-\rho\left[S\left(x^{k}, y^{k}\right)-S\left(x^{*}, y^{*}\right)\right]\right\| \\
\leq & \left(1-a^{k}\right)\left\|g\left(x^{k}\right)-g\left(x^{*}\right)\right\|+a^{k}\left\|g\left(x^{k}\right)-g\left(x^{*}\right)-\rho\left[S\left(x^{k}, y^{k}\right)-S\left(x^{*}, y^{k}\right)\right]\right\| \\
& +a^{k} \rho\left\|S\left(x^{*}, y^{k}\right)-S\left(x^{*}, y^{*}\right)\right\| .
\end{aligned}
$$

Since $S$ is relaxed $(g, \gamma, r)$-pseudococoercive and $(g, \mu)$-Lipschitz continuous in the first variable, and by (iv) we have

$$
\begin{aligned}
\| g\left(x^{k}\right) & -g\left(x^{*}\right)-\rho\left[S\left(x^{k}, y^{k}\right)-S\left(x^{*}, y^{k}\right)\right] \|^{2} \\
= & \left\|g\left(x^{k}\right)-g\left(x^{*}\right)\right\|^{2}-2 \rho\left\langle S\left(x^{k}, y^{k}\right)-S\left(x^{*}, y^{k}\right), g\left(x^{k}\right)-g\left(x^{*}\right)\right\rangle \\
& +\rho^{2}\left\|S\left(x^{k}, y^{k}\right)-S\left(x^{*}, y^{k}\right)\right\|^{2} \\
= & \left\|g\left(x^{k}\right)-g\left(x^{*}\right)\right\|^{2}-2 \rho\left\langle S\left(x^{k}, y^{k}\right), g\left(x^{k}\right)-g\left(x^{*}\right)\right\rangle \\
& +2 \rho\left\langle S\left(x^{*}, y^{k}\right), g\left(x^{k}\right)-g\left(x^{*}\right)\right\rangle+r^{2}\left\|S\left(x^{k}, y^{k}\right)-S\left(x^{*}, y^{k}\right)\right\|^{2} \\
\leq & \left\|g\left(x^{k}\right)-g\left(x^{*}\right)\right\|^{2}+2 \rho \gamma\left\|S\left(x^{k}, y^{k}\right)-S\left(x^{*}, y^{k}\right)\right\|^{2} \\
& +\left(\rho^{2} \mu^{2}\right)\left\|g\left(x^{k}\right)-g\left(x^{*}\right)\right\|^{2}-2 \rho r\left\|g\left(x^{k}\right)-g\left(x^{*}\right)\right\|^{2} \\
& +2 \rho\left\langle S\left(x^{*}, y^{k}\right), g\left(x^{k}\right)-g\left(x^{*}\right)\right\rangle .
\end{aligned}
$$

Since

$$
\begin{gathered}
2\left\langle S\left(x^{*}, y^{k}\right), g\left(x^{k}\right)-g\left(x^{*}\right)\right\rangle \leq\left[\left\|S\left(x^{*}, y^{k}\right)\right\|^{2}+\left\|g\left(x^{k}\right)-g\left(x^{*}\right)\right\|^{2}\right], \\
2\left\|S\left(x^{*}, y^{k}\right)\right\|^{2} \leq\left\|S\left(x^{*}, y^{k}\right)-S\left(x^{k}, y^{k}\right)\right\|^{2} \\
\text { for } 2\left\|S\left(x^{k}, y^{k}\right)\right\|^{2}-\left\|S\left(x^{*}, y^{k}\right)+S\left(x^{k}, y^{k}\right)\right\|^{2}>0
\end{gathered}
$$

and

$$
\left\|S\left(x^{*}, y^{k}\right)\right\|^{2} \leq\left(\mu^{2} / 2\right)\left\|g\left(x^{k}\right)-g\left(x^{*}\right)\right\|^{2}
$$

we have

$$
2 \rho\left\langle S\left(x^{*}, y^{k}\right), g\left(x^{k}\right)-g\left(x^{*}\right)\right\rangle \leq\left[\rho+\left(\rho \mu^{2} / 2\right)\right]\left\|g\left(x^{k}\right)-g\left(x^{*}\right)\right\|^{2} .
$$

It follows that

$$
\begin{aligned}
& \left\|g\left(x^{k}\right)-g\left(x^{*}\right)-\rho\left[S\left(x^{k}, y^{k}\right)-S\left(x^{*}, y^{k}\right)\right]\right\|^{2} \\
& \quad \leq\left\|g\left(x^{k}\right)-g\left(x^{*}\right)\right\|^{2}-2 \rho r\left\|g\left(x^{k}\right)-g\left(x^{*}\right)\right\|^{2}+2 \rho \gamma \mu^{2}\left\|g\left(x^{k}\right)-g\left(x^{*}\right)\right\|^{2}
\end{aligned}
$$




$$
\begin{aligned}
& +(\rho \mu)^{2}\left\|g\left(x^{k}\right)-g\left(x^{*}\right)\right\|^{2}+\left[\rho+\left(\rho \mu^{2} / 2\right)\right]\left\|g\left(x^{k}\right)-g\left(x^{*}\right)\right\|^{2} \\
= & {\left[1-2 \rho r+2 \rho \gamma \mu^{2}+\rho+\left(\rho \mu^{2} / 2\right)+(\rho \mu)^{2}\right]\left\|g\left(x^{k}\right)-g\left(x^{*}\right)\right\|^{2} . }
\end{aligned}
$$

As a result, we have

$$
\begin{aligned}
\| g\left(x^{k+1}\right) & -g\left(x^{*}\right)\left\|\leq\left(1-a^{k}\right)\right\| g\left(x^{k}\right)-g\left(x^{*}\right) \| \\
& +a^{k} \theta\left\|g\left(x^{k}\right)-g\left(x^{*}\right)\right\|+a^{k} \rho \nu\left\|h\left(y^{k}\right)-h\left(y^{*}\right)\right\|,
\end{aligned}
$$

where $\theta=\left[1-2 \rho r+2 \rho \gamma \mu^{2}+\rho+\left(\rho \mu^{2} / 2\right)+(\rho \mu)^{2}\right]^{1 / 2}$.

Similarly, we have

$$
\begin{aligned}
\| h\left(y^{k+1}\right) & -h\left(y^{*}\right)\left\|\leq\left(1-a^{k}\right)\right\| h\left(y^{k}\right)-h\left(y^{*}\right) \| \\
& +a^{k} \sigma\left\|h\left(y^{k}\right)-h\left(y^{*}\right)\right\|+a^{k} \eta \tau\left\|g\left(x^{k}\right)-g\left(x^{*}\right)\right\|,
\end{aligned}
$$

where $\sigma=\left[1-2 \eta s+2 \eta \lambda \beta+\eta+\left(\eta \beta^{2} / 2\right)+(\eta \beta)^{2}\right]^{1 / 2}$.

It follows from (2.2) and (2.3) that

$$
\begin{aligned}
\| g\left(x^{k+1}\right) & -g\left(x^{*}\right)\|+\| h\left(y^{k+1}\right)-h\left(y^{*}\right) \| \\
\leq & \left(1-a^{k}\right)\left\|g\left(x^{k}\right)-g\left(x^{*}\right)\right\|+a^{k} \delta\left\|g\left(x^{k}\right)-g\left(x^{*}\right)\right\| \\
& +\left(1-a^{k}\right)\left\|h\left(y^{k}\right)-h\left(y^{*}\right)\right\|+a^{k} \delta\left\|h\left(y^{k}\right)-h\left(y^{*}\right)\right\| \\
= & {\left[1-(1-\delta) a^{k}\right]\left(\left\|g\left(x^{k}\right)-g\left(x^{*}\right)\right\|+\left\|h\left(y^{k}\right)-h\left(y^{*}\right)\right\|\right) } \\
\leq & \prod_{j=0}^{k}\left[1-(1-\delta) a^{j}\right]\left(\left\|g\left(x^{0}\right)-g\left(x^{*}\right)\right\|+\left\|h\left(y^{0}\right)-h\left(y^{*}\right)\right\|\right),
\end{aligned}
$$

where $d=\max \{\theta+\eta \tau, \sigma+\rho \nu\}$ and $\theta+\eta \tau, \sigma+\rho \nu<1$.

Since $\delta<1$ and $\sum_{k=0}^{\infty} a^{k}$ is divergent, we have from [10] that

$$
\lim _{k \rightarrow \infty} \prod_{j=0}^{k}\left[1-(1-\delta) a^{j}\right]=0
$$

Therefore we have

$$
\left\|g\left(x^{k+1}\right)-g\left(x^{*}\right)\right\|+\left\|h\left(y^{k+1}\right)-h\left(y^{*}\right)\right\| \rightarrow 0 \quad \text { as } \quad k \rightarrow \infty .
$$

Consequently, the sequence $\left\{\left(g\left(x^{k}\right), h\left(y^{k}\right)\right)\right\}$ converges strongly to $\left(g\left(x^{*}\right), h\left(y^{*}\right)\right)$, a solution to the SNVI (1.1)-(1.2). This, in turn, using the expansiveness of $g$ and $h$, implies that sequences $\left\{x^{k}\right\}$ and $\left\{y^{k}\right\}$ converge, respectively, to $x^{*}$ and $y^{*}$ for $\delta<1$.

Corollary 2.2. Let $H_{1}$ and $H_{2}$ be two real Hilbert spaces and $K_{1}$ and $K_{2}$ be nonempty closed convex subsets of $H_{1}$ and $H_{2}$, respectively. Let $S: K_{1} \times K_{2} \rightarrow$ $H_{1}$ be relaxed $(g, \gamma, r)$-cocoercive and $(g, \mu)$-Lipschitz continuous in the first variable, and let $S$ be $(h, \nu)$-Lipschitz continuous in the second variable. Let $T: K_{1} \times K_{2} \rightarrow H_{2}$ be relaxed $(h, \lambda, s)$-cocoercive and $(h, \beta)$-Lipschtz continuous in the second variable, and let $T$ be $(g, \tau)$-Lipschitz continuous in the first 
variable. Let $g: K_{1} \rightarrow K_{1}$ and $h: K_{2} \rightarrow K_{2}$ be expansive mappings. Let $\|\cdot\|_{x}$ denote the norm on $H_{1} \times H_{2}$ defined by

$$
\|(u, v)\|_{x}=\|u\|+\|v\| \quad \text { for } \quad(u, v) \in H_{1} \times H_{2}
$$

where $\|\cdot\|$ denotes the norm on both $H_{1}$ and $H_{2}$.

In addition, let $\delta=\max \{\theta+\eta \tau, \sigma+\rho \nu\}$, where

$$
\begin{gathered}
\theta=\left[1-2 \rho r+2 \rho \gamma \mu^{2}+(\rho \mu)^{2}\right]^{1 / 2}, \\
\sigma=\left[1-2 \eta s+2 \eta \lambda \beta^{2}+(\eta \beta)^{2}\right]^{1 / 2}, \\
\theta+\eta \tau<1, \quad \sigma+\rho \nu<1 .
\end{gathered}
$$

Suppose that the following assumptions hold:

(i) the element $\left(g\left(x^{*}\right), h\left(y^{*}\right)\right) \in K_{1} \times K_{2}$ forms a solution to the SNVI (1.1)-(1.2);

(ii) sequences $\left\{g\left(x^{k}\right)\right\}$ and $\left\{h\left(y^{k}\right)\right\}$ are generated by Algorithm 2.1;

(iii) the sequence $\left\{a^{k}\right\}$ satisfies

$$
0 \leq a^{k} \leq 1 \quad \text { and } \quad \sum_{k=0}^{\infty} a^{k}=\infty .
$$

Then the sequence $\left\{\left(g\left(x^{k}\right), h\left(y^{k}\right)\right)\right\}$ converges to $\left(g\left(x^{*}\right), h\left(y^{*}\right)\right)$.

Corollary 2.3. Let $H_{1}$ and $H_{2}$ be two real Hilbert spaces and $K_{1}$ and $K_{2}$ be nonempty closed convex subsets of $H_{1}$ and $H_{2}$, respectively. Let $S: K_{1} \times$ $K_{2} \rightarrow H_{1}$ be $(g, r)$-strongly pseudomonotone and $(g, \mu)$-Lipschitz continuous in the first variable, and let $S$ be $(h, \nu)$-Lipschitz continuous in the second variable. Let $T: K_{1} \times K_{2} \rightarrow H_{2}$ be $(h, s)$-strongly pseudomonotone and $(h, \beta)$-Lipschitz continuous in the second variable, and let $T$ be $(g, \tau)$-Lipschitz continuous in the first variable. Let $g: K_{1} \rightarrow K_{1}$ and $h: K_{2} \rightarrow K_{2}$ be expansive mappings. Let $\|\cdot\|_{x}$ denote the norm on $H_{1} \times H_{2}$ defined by

$$
\|(u, v)\|_{x}=\|u\|+\|v\| \quad \text { for }(u, v) \in H_{1} \times H_{2},
$$

where $\|\cdot\|$ denotes the norm on both $H_{1}$ and $H_{2}$.

In addition, let $\delta=\max \{\theta+\eta \tau, \sigma+\rho \nu\}$, where

$$
\begin{aligned}
& \theta=\left[1-2 \rho r+\rho+\left(\rho \mu^{2} / 2\right)+(\rho \mu)^{2}\right]^{1 / 2}, \\
& \sigma=\left[1-2 \eta s+\eta+\left(\eta \beta^{2} / 2\right)+(\eta \beta)^{2}\right]^{1 / 2}, \\
& \theta+\eta \tau<1, \quad \sigma+\rho \nu<1 .
\end{aligned}
$$

Suppose that the following assumptions hold:

(i) the element $\left(g\left(x^{*}\right), h\left(y^{*}\right)\right) \in K_{1} \times K_{2}$ forms a solution to the SNVI (1.1)-(1.2) problem;

(ii) sequences $\left\{g\left(x^{k}\right)\right\}$ and $\left\{h\left(y^{k}\right)\right\}$ are generated by Algorithm 2.1;

(iii) the sequence $\left\{a^{k}\right\}$ satisfies

$$
0 \leq a^{k} \leq 1 \quad \text { and } \quad \sum_{k=0}^{\infty} a^{k}=\infty
$$

(iv) $\left\langle S\left(x^{*}, y^{k}\right), g\left(x^{k}\right)-g\left(x^{*}\right)\right\rangle \geq 0$; 
(v) $\left\langle T\left(x^{k}, y^{*}\right), h\left(y^{k}\right)-h\left(y^{*}\right)\right\rangle \geq 0$.

Then the sequence $\left\{\left(g\left(x^{k}\right), h\left(y^{k}\right)\right)\right\}$ converges to $\left(g\left(x^{*}\right), h\left(y^{*}\right)\right)$.

\section{REFERENCES}

1. J. C. Dunn, Convexity, monotonicity, and gradient processes in Hilbert space. J. Math. Anal. Appl. 53(1976), No. 1, 145-158.

2. Y. P. FAng and N. J. HuAng, $H$-monotone operators and system of variational inclusions. Comm. Appl. Nonlinear Anal. 11(2004), No. 1, 93-101.

3. G. Kassay and J. Kolumban, System of multi-valued variational inequalities. Publ. Math. Debrecen 56(2000), No. 1-2, 185-195.

4. D. Kinderlehrer and G. Stampacchia, An introduction to variational inequalities and their applications. Pure and Applied Mathematics, 88. Academic Press, Inc. [Harcourt Brace Jovanovich, Publishers], New York-London, 1980.

5. B. E. RhoAdes and R. U. Verma, Two-step algorithms and their applications to variational problems. Comm. Appl. Nonlinear Anal. 11(2004), No. 2, 45-55.

6. R. U. VERMA, Projection methods, algorithms, and a new system of nonlinear variational inequalities. Comput. Math. Appl. 41(2001), No. 7-8, 1025-1031.

7. R. U. VERMA, A new class of iterative algorithms for approximation-solvability of nonlinear variational inequalities. Comput. Math. Appl. 41(2001), No. 3-4, 505-512.

8. R. U. Verma, Role of generalized partial pseudomonotonicity in solvability of nonlinear variational inequalities. Int. J. Comput. Numer. Anal. Appl. 3(2003), No. 2, 125-132.

9. R. U. Verma, Nonlinear variational and constrained hemivariational inequalities involving relaxed operators. Z. Angew. Math. Mech. 77(1997), No. 5, 387-391.

10. R. Wittmann, Approximation of fixed points of nonexpansive mappings. Arch. Math. (Basel) 58(1992), No. 5, 486-491.

11. N. H. XiU and J. Z. ZhANG, Local convergence analysis of projection-type algorithms: unified approach. Dedicated to Professor Wolfram Stadler. J. Optim. Theory Appl. 115(2002), No. 1, 211-230.

12. E. ZeIdLER, Nonlinear functional analysis and its applications. II/A. Linear monotone operators. (Translated from the German) Springer-Verlag, New York, 1990.

(Received 14.04.2004)

Authors' addresses:

Ravi P. Agarwal

Florida Institute of Technology

Department of Mathematics

Melbourne, Florida 32901, USA;

E-mail: agarwal@fit.edu

Donal O'Regan

National University of Ireland

Department of Mathematics

Galway, Ireland;

E-mail: donal.oregan@nuigalway.ie 
Ram U. Verma

University of Toledo

Department of Mathematics

Toledo, Ohio 43606, USA

E-mail: verma99@msn.com 